ISSN: 2539-3804@®@@

\title{
La definitud en el sintagma nominal en wayunaiki ${ }^{1}$
}

\author{
Rudecindo Ramírez González² \\ Universidad de la Guajira \\ Riohacha, Colombia
}

\section{Resumen}

El presente artículo describe la categoría de definitud en wayunaiki (Arawak Septentrional-: Colombia). Se propone que en esta lengua el sintagma nominal contiene elementos gramaticales cuyo significado es el de definido o indefinido. La categoría de definitud se marca en los nombres, en los artículos y en los adjetivos. El indefinido (wanee) es léxico y el definido (-ka-) es sufijo. En una primera parte se presenta el estado del arte correspondiente al fenómeno. En una segunda parte, mostraremos nuestros propios resultados comparados con datos de otros autores. Finalmente, en una tercera parte, presentaremos nuestras conclusiones sobre este fenómeno.

Palabras clave: definitud; wayunaiki; lenguas amerindias; descripción lingüística; morfología.

\footnotetext{
${ }^{1}$ Este artículo presenta los hallazgos sobre definitud en el marco de la investigación "Categorías gramaticales relacionadas con el nombre en wayunaiki" conducente a la tesis doctoral del autor para el Doctorado en Lingüística de la Universidad de Antioquia, Medellín, Colombia.

${ }^{2}$ El autor agradece a los informantes Griselda Iguarán, Mauricia Pushaina, Nestor Epieyu, Anibal Epieyu y Blanca Epieyu, en La Guajira, que lo apoyaron con los datos lingüísticos para realizar el análisis presentado. Así mismo, agradece a la Universidad de la Guajira y la Gobernación por la beca Contrato de Crédito Educativo Condonable PFAN 006 de 2014, otorgada para llevar a cabo la investigación de la cual se presenta una parte.
} 


\section{Abstract \\ Definiteness in the noun phrase in wayunaiki}

The present article describes the category of definiteness in Wayunaiki (Septentrional- Arawak: Colombia). The literature suggests that the noun phrase in this language has grammatical elements that lend it as either definite or indefinite. The definiteness category is marked on nouns, articles and adjectives. The indefinite (wanee) is lexical and the definite $(-k a-)$ is a suffix. The first part of the paper presents the state of the art regarding this phenomenon. The second part shows the findings of the current study compared to previous data from other authors. Finally, in the third part, we present our conclusions regarding this phenomenon.

Key words: definiteness; Wayunaiki; Amerindian languages; linguistic description; morphology.

\section{Résumé}

\section{La définitude dans le syntagme nominal en wayunaiki}

Cet article décrit la catégorie de définitude en wayunaiki (Arawak -Septentrional-: Colombie). On affirme que dans cette langue le syntagme nominal contient des éléments grammaticaux dont le sens est défini ou indéfini. La définitude se trouve marquée dans les noms, les articles et les adjectifs. L'indéfini (wanee) est lexical et le défini (-ka-) est un suffixe. Dans un premier temps, nous présenterons l'état de l'art concernant à ce phénomène. Ensuite, nous montrerons nos propres résultats comparés à ceux des autres auteurs. Pour terminer, nous présenterons nos conclusions sur ce phénomène.

Mots-clés : définitude ; wayunaiki ; langues Amérindiennes ; description linguistique; morphologie. 


\section{Cómo CITAR ESTE ARTÍCULO}

Ramírez González, R. (2019). La definitud en el sintagma nominal en wayunaiki. Lenguaje, 47(1), 28-45. doi: 10.25100/lenguaje.v47i1.7182 


\section{INTRODUCCIÓN}

En el Departamento de La Guajira, Colombia, se habla la lengua wayunaiki ${ }^{3}$ en las regiones Alta, Media y Baja Guajira de la Península del mismo nombre, desde la costa de Punta Espada hasta la costa del municipio de Camarones, desde la Serranía de Macuira hasta las estribaciones de la Sierra Nevada de Santa Marta y desde el desierto de Taroa y Punta Gallina hasta el bosque tropical de Aremasain y Carraipia. En realidad no se sabe a ciencia cierta cuántos wayu hablantes hay en la actualidad. ${ }^{4}$ También hay un gran número de hablantes en el Estado Zulia en Venezuela, que pueden superar los ciento cincuenta mil (Landaburu, 2005). El wayunaiki pertenece a la familia lingüística Arawak y se subclasifica en la rama Arawak Septentrional o Norteña (ver p. ej. Aikhenvald, 1999 y Landaburu, 2005). La lengua wayunaiki se encuentra geográficamente aislada de las demás lenguas Arawak, cuyos hablantes se encuentran principalmente en la región de la Amazonía y los llanos orientales.

Es una lengua de tradición oral, y aún es generalmente transmitida por los adultos a las nuevas generaciones. En el ámbito regional, el wayunaiki es, con el español, la lengua cooficial en el territorio del Departamento de La Guajira.

Como es el caso para muchas lenguas indígenas colombianas, aunque ya existen diversos estudios sobre algunos aspectos de la gramática del wayunaiki (Ehrman, 1972; Goulet y Jusayú, 1978; Mansen y Captain, 2000; Mansen y Mansen, 1984; Olza y Jusayú, 2012; Pérez, 1986; Ramírez, 1995), aún es mucho el camino por recorrer para llegar a una descripción adecuada de su estructura en todos los niveles. En el marco de una investigación sobre la morfología del nombre y categorías asociadas en esta lengua, presentamos a continuación una propuesta de análisis de la expresión morfológica de la categoría de la definitud, que, junto con las de género, número y caso, constituyen las principales categorías gramaticales que se suelen expresar en el nombre y sus modificadores en las lenguas naturales. Después de una breve definición teórica general del concepto de definitud que sirvió de fundamento para el análisis, de una síntesis de los resultados de trabajos anteriores sobre el tema, y de una descripción de la metodología empleada en la investigación, se propone un análisis alternativo (aunque también enmarcado dentro de la lingüística descriptiva) de las marcas morfológicas de definitud en el wayunaiki.

3 En la literatura sobre esta lengua se usan diferentes términos para denominarla: guajiro, wayuunaiki, wayuu. En el presente artículo haré uso del término wayunaiki.

${ }^{4}$ Pero se tiene la información de que la población wayu en Colombia es de 270.413 (DANE, 2005). 


\section{LA CATEGORÍA DE DEFINITUD}

La definitud en cuanto categoría semántica y pragmática es compleja y presenta no pocas dificultades a la hora de su definición. La siguiente, propuesta por Givón (1984), puede servir de guía, pero no es incontrovertida:

Los hablantes codifican una construcción nominal como indefinida si piensan que no están autorizados a asumir que el oyente pueda -por cualesquiera medios-asignarle a aquella una única identidad referencial. Los hablantes codifican una construcción nominal referencial como definida si piensan que están autorizados a asumir que el oyente puede -por cualesquiera medios- asignarle a aquella una única referencia. (p. 398).

Basta con exponer los ejemplos siguientes para ver que la definición anterior (basada en el concepto de la unicidad o de la identificabilidad) no es completamente satisfactoria, como no lo son tampoco, por cierto, las definiciones basadas en otros conceptos como el de la inclusividad:

(1) El que te ganaste es un premio muy merecido.

(2) El compañero que salga de último, cierra la puerta, por favor.

(3) Estamos buscando a los irresponsables que se llevaron las llaves.

No se presentará aquí por razones de espacio la discusión que aún perdura acerca de los conceptos de identificabilidad o inclusividad subyacentes a la definitud semántico-pragmática. Una exposición detallada del estado de la discusión a este respecto se encuentra en Lyons (1999).

Para el presente análisis partiremos de la categoría gramatical de definitud, siguiendo la diferenciación que propone Lyons (1999):

(...) there is a distinction to be made between grammatical definiteness and semantic/pragmatic definiteness, and on the concept of grammaticalization. The proposal is that definiteness stricto sensu is not a semantic or pragmatic notion as assumed by almost all writers on the subject, but rather a grammatical category on a par with tense, mood, number, gender etc. But, like these, it is the grammaticalization (that is, the representation in grammar) of some category of meaning. (pp. 274-275).

El rasgo [+DEF] o [-DEF] puede estar contenido inherentemente en diferentes clases de palabras y tipos de construcciones, como por ejemplo en 
pronombres demostrativos y personales, construcciones posesivas, cuantificadores y nombres propios. De manera explícita, en unidades gramaticales, se pueden expresar a través de unidades léxicas como los artículos que generalmente son clíticos o ligados. En este sentido, Creissels (2006) afirma que:

Beaucoup de langues appartenant aux familles les plus diverses ont un 'article défini'. Bien que souvent écrits comme des mots à part, les articles définis sont généralement des formes liées, mais attachées selon les langues au premier ou au dernier mot du constituant nominal, et pas forcément au nom. (p. 56).

Un ejemplo de artículos que se escriben separadamente, como si fueran palabras independientes, pero que en realidad son clíticos, son los artículos definidos del español (Leonetti, 1999). ${ }^{5}$

Un ejemplo de artículos ligados se encuentra en el árabe. Como se ve en los ejemplos de (4a), el artículo definido presenta un fenómeno de asimilación que muestra su naturaleza ligada. La marca de definitud puede dar lugar a una concordancia entre el sustantivo y algunos de sus dependientes, como lo ilustra

33 Creissels igualmente a través del árabe, en el ejemplo (4b) (los ejemplos son de Creissels, 2006, p. 56):

(4) a. al-bajtu 'la casa'

al-kitābu 'el libro'

as - -amsu 'el sol'

ar-razulu 'el hombre'

an-nāru 'el fuego'

$\begin{array}{cl}\text { b. bajtu- } \boldsymbol{n} \quad \text { kabìru- } \boldsymbol{n} & \text { al-bajtu } \quad \boldsymbol{l} \text {-kabìru } \\ \text { casa-INDEF grande-INDEF } & \text { DEF-casa DEF-grande } \\ \text { 'una casa grande' } & \text { 'la casa grande' }\end{array}$

En el subcapítulo siguiente se presentan las propuestas de análisis de la categoría gramatical de la definitud en wayunaiki en trabajos anteriores.

\footnotetext{
5 "Tanto las formas de [artículo definido] masculino y femenino como la forma lo se caracterizan por ser átonas y funcionar como clíticos, es decir, por apoyarse fónicamente en el constituyente que las sigue." Otro rasgo que permite clasificarlos como clíticos es "(...) la falta de independencia sintáctica: los artículos no pueden constituir SSNN por sí mismos, y deben ir incluidos en un sintagma en el que aparezca algún elemento léxico (habitualmente un nombre, pero no necesariamente) del que sean proclíticos." (Ambas citas provienen de Leonetti, 1999, p. 791).
} 


\section{ESTUDIOS ANTERIORES SOBRE LA DEFINITUD EN WAYUNAIKI}

Hasta la fecha sigue siendo tema de controversia la definitud en wayunaiki, ya que algunos autores consideran que algunas marcas que expresan esta categoría contienen también simultáneamente otras categorías, como la de género. Este es el caso de Mansen y Mansen (1984), Olza y Jusayú (2012), Pérez (1986), Álvarez (1994), Ehrman (1972) y Goulet y Jusayú (1978), e incluso en trabajos anteriores propios Ramírez (1995).

Mansen y Mansen (1984) consideran los morfemas -kai- y -kat- como artículos definidos de uso opcional, los cuales también contienen información sobre género (masculino y femenino respectivamente) y número. Según estos autores el sufijo - kai "tiene un uso más restringido (...) porque se usa mayormente para referirse a cosas que son expresamente masculinas y estas marcas solamente ocurren en la forma singular" (p. 45). Presentan los siguientes ejemplos:

(5) (Mansen y Mansen 1984, p. 39)
a. kaüshi, -kai
gordo, el
b. miichi, -kat
casa, $1 a^{6}$

Para Olza y Jusayú (2012) los morfemas -kalü, -kát y -ká son sufijos determinativos, femenino singular y $-k a i$ es sufijo determinativo, singular masculino. "El sustantivo masculino va precedido del anunciativo chi y lleva el sufijo especificativo o particularizador -kai":

(6) Olza y Jusayú $(2012$, p. 5)
chi waiúkai el hombre, el guajiro
chi ali'junakai el hombre no indígena
chi pülíkukai el asno, el borrico
chi átpanákai el conejo

\footnotetext{
${ }^{6}$ Las glosas aparecen de esta manera en el texto original.

${ }^{7}$ Los ejemplos en el texto original no se segmentan ni se glosan.
} 
Los sustantivos de género femenino van precedidos del anunciativo tü y seguidos del sufijo especificativo o particularizador -kalü, que se puede apocopar en -ká y en -kát:

(7) Olza y Jusayú (2012, p. 5)

tü músaká la gata

tü jime'kalü el pescado, el pez

tü úchiká el cerro, la montaña

El morfema -kat es el más extendido a muchos nombres, según afirman Olza y Jusayú (2012), “el femenino es en guajiro el archigénero, porque se puede aplicar también a personas y animales machos" (p. 31).

Álvarez (1994) y Ehrman (1972) concuerdan en que un sustantivo adquiere determinación de género a través de los 'sufijos determinativos' -ka -kat -kalü (femenino singular), -kai -kali (masculino singular) y -kana (plural), que habitualmente co-ocurren formando una determinación o determinante con el demostrativo chi (masculino) y tü (femenino). Para Ehrman (1972), chi y tü son demostrativos de mayor proximidad con "rol semántico" de artículo.

\section{Metodología}

La presente investigación se realizó con base en datos recogidos en terreno con hablantes bilingües (wayunaiki-español) de la comunidad de Nazareth y Media Luna en la región de la Alta Guajira, entre 2014 y 2016. Los cuatro informantes tenían entre 29 y 53 años de edad, dos pertenecen a la comunidad de Nazareth y dos a la de Media Luna. Se escogió esta región porque sus hablantes en general se consideran más competentes en wayunaiki que en las demás regiones de la península.

El corpus está conformado por diversos tipos de muestras recolectadas: grabación de narraciones y diálogos espontáneos, así como cuestionarios diseñados para la elicitación de los fenómenos específicos estudiados. En el caso de los cuestionarios se les pidió a los hablantes la traducción oral (y en algunos casos escrita) del español al wayunaiki de estructuras que podrían contener expresiones de definitud, es decir, nombres de diversos tipos con sus categorías asociadas. Uno de los cuestionarios contenía sintagmas nominales definidos e indefinidos, y el otro cuestionario contenía oraciones transitivas e intransitivas con sintagmas nominales definidos e indefinidos. 
Los datos lingüísticos suministrados por los informantes fueron grabados en una grabadora digital con micrófono externo. Para cada cuestionario se registró la fecha, el lugar y los siguientes datos de cada informante: nombre, edad, sexo, escolaridad y oficio. También se recogió el consentimiento de los hablantes para colaborar en la investigación. Todos los textos fueron transcritos por el investigador, que es hablante nativo del wayunaiki, con la asistencia de otros hablantes nativos.

El estudio de los datos se realizó haciendo uso de las categorías de análisis de la Lingüística Descriptiva, en cuyo marco se inscribe esta investigación.

\section{Análisis de la expresión de la definitud en wayunaiki}

Como se dijo anteriormente, en trabajos anteriores de otros lingüistas se analizaban los segmentos -kai y -kat de los ejemplos (5) - (7) como un solo morfema que remite a género, a género y número, o a género y determinante. Hay que señalar que ninguno de estos autores se dedicó al tema de la morfología nominal en profundidad.

$\mathrm{Al}$ analizar con detalle los datos del corpus recogido para la presente investigación, enfocada en la morfología del nombre, es evidente que -kat y -kai deben contener no solo género sino también definitud, ya que en (8) y (9), ejemplos en los que el sintagma nominal en cuestión tiene sentido definido, aparecen -kat y $k a i$, y en (10) y (11), que contrastan con los anteriores únicamente en cuanto al sentido indefinido, no aparecen estas formas:

$\begin{array}{lll}\text { ant-ü-shi aliika } & \text { chi } & \text { e'iyoou-kai } \\ \text { llegar-PAS-MASC.SG ayer } & \text { DEF.MASC.SG } & \text { visitante-DEF.MASC.SG } \\ \text { 'ayer llegó el visitante' } & & \end{array}$

ant-ü-sü aliika tü e'iyoou-kat

llegar-PAS-FEM.SG ayer DEF.FEM.SG visitante-DEF.FEM.SG 'ayer llegó la visitante'

\begin{tabular}{|c|c|}
\hline aliika-müin & wanee-(shia $)^{8}$ \\
\hline $\begin{array}{l}\text { llegar-PAS-MASC.SG ayer-DAT } \\
\text { ‘ayer llegó un visitante’ }\end{array}$ & INDEF.SG(-MASC) \\
\hline
\end{tabular}

${ }^{8}$ Esta marca de género es opcional. Parece ser que esta marca se usaba con mayor frecuencia entre las generaciones anteriores, y que hoy en día se tiende a elidir. 


$\begin{array}{llll}\text { ant-ü-sür aliika-müin } & \text { wanee-(sia) } & \text { e'iyoou } \\ \text { llegar-PAS-FEM.SG } & \text { ayer-DAT } & \text { INDEF-FEM } & \text { visitante } \\ \text { 'ayer llegó una visitante' } & & \end{array}$

Es decir, -kat y -kai contienen el significado de 'definido' mientras que wanee(-shia) y wanee(-sia) contienen el significado de 'indefinido'. Obsérvense los ejemplos (12) y (13) con -kana y con -kalü-irua para ver el contraste entre las formas que expresan definido singular -ver (8) y (9)- y definido plural:

ant-ü-shii aliika-müin naa e'iyoou-kana

llegar-PAS-PL ayer-DAT DEF.PL visitante-DEF.MASC.PL

'ayer vinieron los visitantes'
ant-ü-sü-irua
aliika-müin naa
e'yoou-kalü-irua
llegar-PAS-FEM-PL ayer-DAT DEF.PL visitante-DEF.FEM-PL
'ayer vinieron las visitantes' ${ }^{9}$

Una primera propuesta de análisis podría ser entonces considerar los siguientes segmentos como morfemas acumulativos, que expresan al mismo tiempo definitud, género y número:

$$
\begin{aligned}
& -k a i=\text { DEF.MASC.SG } \\
& -k a t=\text { DEF.FEM.SG } \\
& -k a n a=\text { DEF.MASC.PL } \\
& -k a l \ddot{\text { - }} \text { irua }=\text { DEF.FEM-PL }
\end{aligned}
$$

Sin embargo, teniendo en cuenta que el segmento - $k a$ - se encuentra en todas las formas que comparten el significado de $[+\mathrm{DEF}]$ y que está ausente en las formas con interpretación indefinida, se puede proponer otro posible análisis, según el cual estos segmentos constan de dos morfemas diferentes, siendo - $k a$ - el encargado de expresar la definitud y el morfema que le sigue el de expresar el género y el número. Es decir:

$$
\begin{aligned}
& -k a-=\text { DEF } \\
& -k a-i=\text { DEF-MASC.SG }
\end{aligned}
$$

\footnotetext{
${ }^{9}$ El indefinido plural 'unos /unas visitantes' será presentado más adelante.
} 
$-k a-t=$ DEF-FEM.SG

$-k a-n a=$ DEF-MASC.PL

$-k a-l \ddot{u}-i r u a=$ DEF-FEM-PL

El hecho de reencontrar $-i$ y $-t$ (sin $-k a-)$ con la misma forma y el mismo significado (MASC.SG y FEM.SG respectivamente) en otros contextos, como en (14), parece indicar que $-i \mathrm{y}-t$ se pueden segmentar de $-k a-$ :

jintü- $i \quad$ niño

jintü- $t \quad$ niña

jima- $i \quad$ adolescente (hombre)

jimo-t adolescente (mujer)

majayü-t muchacha

En derivaciones nominales:

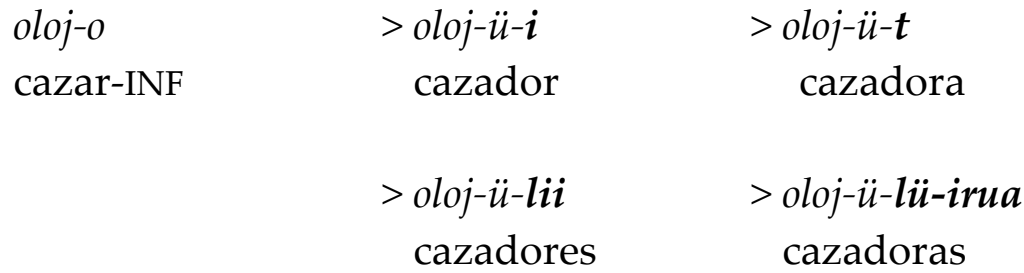

$m a-o^{\prime} u-s a-i$

NEG-ojo-DER-MASC.SG

'ciego' (sin su ojo)

ma-o'u-sa-t

NEG-ojo-DER-FEM.SG

'ciega' (sin su ojo) 
ma-anüi-sa-i

NEG-habla-DER-MASC.SG

'mudo' (sin su lengua)

ma-anüi-sa-t

NEG-habla-DER-FEM.SG

'muda' (sin su lengua)

En pronombres interrogativos:

jarra-i nia?

INTERR-MASC.SG 3SG.MASC

'¿quién es él?'

jarra-t jia?

INTERR-FEM.SG 3SG.FEM

‘quién es ella?'

Se asumirá entonces que - $k a$ - es la marca de definido, y que el morfema siguiente corresponde a la marca de género y número.

Obsérvese que en los sintagmas nominales definidos, como los que aparecen en los ejemplos (8), (9), (12) y (13), aparecen antes del nombre unos determinantes (chi, tü y naa) que establecen concordancia con los sufijos nominales $(-k a-i,-k a-t,-k a-$ na y -ka-lü-irua). Estas unidades se interpretan como determinantes, más específicamente como artículos, que establecen una concordancia con el nombre, ya que repiten la información de definitud y número que aparece en los sufijos nominales, y en las formas singulares, también la de género:

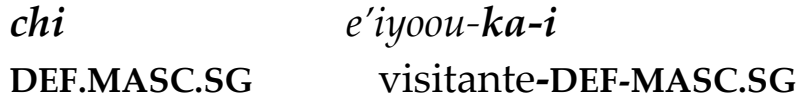
tï $\quad e^{\prime}$ iyoou-ka-t
DEF.FEM.SG visitante-DEF-FEM.SG

naa e'iyoou-ka-na

DEF.PL visitante-DEF-MASC.PL

(19) naa e'yoou-ka-lü-irua

DEF.PL visitante-DEF-FEM-PL 
Como se ve en los ejemplos expuestos, hay una asimetría entre las estructuras de los sintagmas nominales definidos e indefinidos: las marcas de definido van sufijadas al nombre y constituyen morfemas ligados. Además exigen la presencia obligatoria de los artículos, que concuerdan con ellas en cuanto a las categorías de definitud y número (y género en las formas singulares). Las marcas de indefinido, en cambio, son morfemas libres, van antepuestos al nombre, y no exigen concordancia con un determinante.

$\begin{array}{llll}\text { ant-ee-chi wanee- } \varnothing & \text { e'iyou } & \text { joolu'u } \\ \text { llegar-FUT-MASC.SG INDEF-SG } & \text { visitante } & \text { ahora } \\ \text { 'ahora va a llegar un visitante' } & & \end{array}$

$\begin{array}{lll}\text { ant-ee-chi wanee-shia } e^{\prime} \text { iyou joolu'u } & \text { wour } \\ \text { llegar-FUT-MASC.SG INDEF-MASC visitante } & \text { ahora } \\ \text { 'ahora va a llegar un visitante' } & & \end{array}$

ant-ee-rü wanee- $\varnothing$ e'iyou joolu'u

llegar-FUT-FEM.SG INDEF-SG visitante ahora

'ahora va a llegar una visitante'

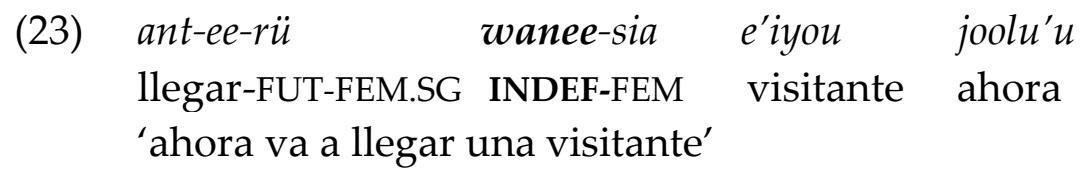

Como se ve en (20) y (21), así como en (22) y (23), wanee puede aparecer sin el sufijo de género. Aquí hay variación, aunque parece haber una tendencia reciente a omitir la marca de género. Esta omisión no tiene consecuencias para la interpretación del género, ya que esta categoría está marcada obligatoriamente en la flexión verbal.

Hay que hacer la aclaración en este punto de que las flexiones verbales de género varían parcialmente en la forma, dependiendo del tiempo y del modo verbal. Es decir, con la marca -ee- de tiempo futuro -ejs. (20), (23)-, aparece -chi como marca de masculino y -rü como marca de femenino, mientras que, con el tiempo pasado, aparece -shi como marca de masculino y -sü como marca de femenino -ejs. (8), (11)-. La marca de género en el verbo es importante también porque ayuda, entre otras cosas, a desambiguar el significado de -ee-, que puede ser marca de tiempo futuro o marca de modo desiderativo, según si va seguido de -chi (futuro) o de -shi (desiderativo) en el masculino, y de -rü (futuro) o -sü (desiderativo), en el femenino (ver Ramírez, 1995). 
A continuación, se contrastan los ejemplos anteriores con las formas del plural:

ant-ee-nü wanee-irua e'iyou joolu'u llegar-FUT-PL INDEF-PL visitante ahora 'ahora van a llegar unos visitantes'

$\begin{array}{llll}\text { ant-ee-nü } & \text { wanee-shia-irua } & e^{\prime} i y o u & \text { joolu'u } \\ \text { llegar-FUT-PL } & \text { INDEF-MASC-PL } & \text { visitante } & \text { ahora }\end{array}$
'ahora van a llegar unos visitantes'

ant-ee-nü wanee-sia-irua e'iyou joolu'u llegar-FUT-PL INDEF-FEM-PL visitante ahora 'ahora van a llegar unas visitantes'
ant- ̈u-shii
wanee-iru
e'iyou
joolu'u
llegar-PAS-PL INDEF-PL visitante ahora
'ahora llegaron unos visitantes'

$\begin{array}{llll}\text { ant-ü-sü-irua wanee-irua } & e^{\prime} i y o u & \text { joolu'u } \\ \text { llegar-PAS-FEM-PL INDEF-PL visitante } & \text { ahora } \\ \text { 'ahora llegaron unas visitantes' } & \end{array}$
ant-ü-sü-irua
wanee-sia-irua e'iyou
joolu'u

llegar-PAS-FEM-PL INDEF-FEM-PL visitante ahora 'ahora llegaron unas visitantes'

Como se ve en (24), (25) y (26), para marcar el indefinido plural puede aparecer la marca de plural -irua sola o acompañada de la marca de género -shia(masculino) o-sia- (femenino).

En cuanto a las marcas de concordancia con el sujeto en el verbo, obsérvese el contraste de (24) - (26) con (27) - (29), del cual se desprende que la forma de la marca de número en el verbo varía según el tiempo: con la marca de futuro -eeaparece la marca de plural -nü para marcar la concordancia verbal con el sujeto, así este sea femenino o masculino. Y con la marca de pasado - ̈̈- se usa -shii como marca de número plural (el género puede ser masculino o femenino) y -sü-irua para marcar la concordancia de femenino y plural, respectivamente.

Los adjetivos también pueden llevar marcas de definido cuando modifican un nombre dentro de un sintagma nominal, como se ve en los ejemplos (30) y (31). 
(30) atunk-ü-shi chi alijuna shukula-ka-i

dormir-PAS-MASC.SG DEF.MASC.SG criollo flojo-DEF-MASC.SG

'el criollo flojo se durmió'

(31) o'un- ̈ü-sü tü jiet anamia-ka-t ir-PAS-FEM.SG DEF.FEM mujer amable-DEF-FEM.SG

'la mujer amable se fue'

Algunos adjetivos en construcciones predicativas permanecen invariables, o sea que no van seguidos por el sufijo de género/número, ni van marcados para definitud, como se ve en los siguientes ejemplos:

$\begin{array}{llll}\text { mulo'u } & n \ddot{u} \text {-asii } & k a^{\prime} u l a-k a-i & c h i \\ \text { largo } & \text { 3SG.MASC-rabo } & \text { chivo-DEF-MASC.SG } & \text { DEM.MASC.SG }\end{array}$

'el rabo de este chivo es largo'

(33) jutpüna main Piirai nulia Maliwou

alto más Piirai que Maliwou

'Piirai es más alto que Maliwou'

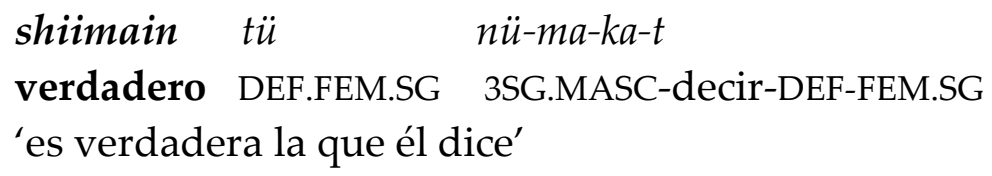

\section{CONCLUSIONES}

En conclusión, la categoría de la definitud se marca en wayunaiki en los nombres, en los artículos y en los adjetivos, cuando estos últimos son atributivos. En nuestros datos se encontró que la categoría de definitud asociada al nombre se marca gramaticalmente de la siguiente manera: el valor 'definido' se expresa a través del sufijo $-k a-$, al cual le siguen las marcas de género y número. El valor 'indefinido' se expresa por medio del morfema libre wanee, que se antepone al nombre, y al cual se le pueden sufijar marcas de género y número. Los artículos con el significado de 'definido' (chi, tü y naa) aparecen antes del nombre y establecen concordancia en cuanto a las categorías de definitud y número (y género en las formas singulares) con los sufijos nominales (-ka-i, -ka-t, -ka-na y -kalü-irua), como se muestra en la Tabla 1 : 
Tabla 1. Marcas de definitud con el valor definido

\begin{tabular}{|l|l|l|l|}
\hline \multicolumn{2}{|l|}{ Artículos nominales definidos } & \multicolumn{2}{l|}{ Concordancia con sufijos nominales } \\
\hline DEF.MASC.SG & Chi & DEF-MASC.SG & -ka-i \\
\hline DEF.FEM.SG & tü & DEF-FEM.SG & -ka-t \\
\hline DEF.PL & naa & DEF-MASC.PL & -ka-na \\
& & DEF- FEM - PL & -ka-lü-irua \\
\hline
\end{tabular}

Las marcas de indefinido, en cambio, son morfemas libres, van antepuestos al nombre, y no exigen concordancia con un determinante.

\section{REFERENCIAS}

Aikhenvald, A. (1999). The Arawak language family. En R. M. W. Dixon y A. Aikhenvald (Eds.), The Amazonian Languages (65-106). Cambridge: Cambridge University Press.

Álvarez, J. (1994). Estudios de Lingüística Guajira. Maracaibo, Venezuela: Secretaría de Cultura del Estado Zulia.

Creissels, D. (2006). Syntaxe générale. En Une introduction typologique (Vol. 1 y 2). Paris: Lavoisier.

Ehrman, S. (1972). Wayuunaiki: A Grammar of Guajiro (Tesis doctoral inédita). New York: University of Columbia.

Givón, T. (1984). Syntax: A Functional-Typological Introduction (Vol. I) Amsterdam: John Benjamins.

Goulet, J., y Jusayú, M. (1978). El idioma guajiro; sus fonemas, su ortografía, su morfología. Caracas: Universidad Católica Andrés Bello.

Landaburu, J. (2005). Las lenguas indígenas de Colombia: presentación y estado del arte. Amerindia, (29/30), 3-22. 
Leonetti, M. (1999). El artículo. En I. Bosque y V. Demonte (Coords.), Gramática descriptiva de la lengua española (Vol. 1) (pp. 789-890). Madrid, España: Espasa Calpe.

Lyons, C. (1999). Definiteness. New York: Cambridge University Press.

Mansen, K., y Mansen, R. (1984). Aprendamos Guajiro. Gramática Pedagógica de Guajiro. Bogotá, Colombia: Editorial Townsend.

Mansen, R., y Captain, D. (2000). El idioma wayuu (o guajiro). En M. González de Pérez y M. Rodríguez de Montes (Eds.), Lenguas indígenas de Colombia: una visión descriptiva (pp. 795-810). Bogotá, Colombia: Instituto Caro y Cuervo.

Olza, J., y Jusayú, M. (2012). Gramática de la Lengua Guajira. Caracas, Venezuela; Universidad Católica Andrés Bello.

Pérez van L. F. (1986). El Sintagma Nominal en Wayuunaiki (Tesis de Maestría). Universidad de los Andes, Bogotá, Colombia.

Ramírez, R. (1995). Morfología del verbo en Wayuunaiki (Tesis de Maestría). Universidad de los Andes, Bogotá, Colombia.

\begin{tabular}{|l|l|}
\hline Tabla de abreviaturas & \\
\hline 3SG & Tercera persona singular \\
DAT & Dativo \\
DEF & Definido \\
DER & Derivativo \\
FEM & Femenino \\
FUT & Futuro \\
INDEF & Indefinido \\
INF & Inflexión \\
INTERR & Interrogativo \\
MASC & Masculino \\
NEG & Negativo \\
PAS & Pasado \\
PL & Plural \\
SG & Singular \\
\hline
\end{tabular}




\section{SOBRE EL AUTOR}

\section{Rudecindo Ramírez González}

Candidato a doctor en Lingüística de la Universidad de Antioquia, Colombia; Magíster en Etnolingüística de la Universidad de los Andes de Bogotá; profesor titular adscrito a la Facultad de Ciencias de la Educación de la Universidad de La Guajira, Colombia; docente del área de la Lingüística teórica y descriptiva y miembro del grupo de investigación de sociolingüística GES de la Universidad de Antioquia.

Correo electrónico: rramirez@uniguajira.edu.co 